\title{
Spontaneous Pneumomediastinum: A Rare Radiologic Finding in Covid-19
}

\author{
Swetha Ramireddy* MD, Saumeet Shah MD, Fayzan Ahmad MD, Thomas Bebekoski MD, \\ Anuradha Sreenivasan DO, Kevin Brody MD \\ Henry Ford Macomb Hospital, 1585519 Mile Road, Clinton Township, Michigan, USA 48038
}

*Corresponding Author: Swetha Ramireddy MD, Henry Ford Macomb Hospital, 1585519 Mile Road, Clinton Township, Michigan, USA 48038.

\begin{abstract}
Severe acute respiratory syndrome coronavirus 2 (SARS-CoV-2), and the disease it causes, COVID-19, has been responsible for the death of over 300,000 people globally. Spontaneous pneumomediastinum is an extremely rare radiologic finding of COVID-19, possibly indicating impending intubation or mortality. Here, we present a case series of two patients with COVID-19, who were found to have pneumomediastinum on imaging.
\end{abstract}

Keywords: spontaneous pneumomediastinum; COVID-19; chest pain; subcutaneous emphysema; dyspnea

\section{INTRODUCTION}

In December of 2019, a novel strain of corona virus was identified to be the cause of a growing-number of cases of pneumonia in Wuhan, China. Severe acute respiratory syndrome coronavirus 2 (SARS-CoV-2), and the disease it causes, termed COVID-19, had turned into a pandemic by March 2020. At the time of writing, approximately 6.8 million people have been confirmed to be infected with SARS-CoV-2 [1]. Common radiological findings of COVID-19 include bilateral lower lobe infiltrates or ground glass opacifications on chest $\mathrm{x}$-ray and chest computerized tomography (CT), respectively $[2,3]$. Here, we present two rare cases of patients with COVID-19 who incidentally had the presence of a pneumomediastinum on imaging.

\section{Case Presentation}

\subsection{Case 1}

A 46-year-old male with a past medical history significant for only marijuana use was admitted with chest pain and shortness of breath. The patient had been experiencing 11 days of nonbloody, watery diarrhea and had been diagnosed with the novel SARS-CoV-2 virus in the outpatient setting three days prior. He denied experiencing a cough or emesis. He had woken up the morning of admission with a sharp substernal chest pain that he noted to be worse with inspiration. Vital signs upon arrival were significant for tachycardia (heart rate of 116) and a temperature of 37.8 Celsius. Physical examination did not reveal crepitus however was remarkable for posterior oropharyngeal erythema. An initial complete blood count (CBC) with differential revealed elevated leukocytes, 11.4, with an absolute neutrophil count of 9.6, while the absolute lymphocyte count of 1.10 was within a normal range. Labs obtained as part of the COVID-19 panel at our institution resulted in a ferritin of 1,088 , creactive protein (CRP) concentration of $22.1, \mathrm{~d}-$ dimer 1.30, and an interleukin 6 (IL-6) of 33.2. Chest (x-ray) done on arrival revealed bilateral infiltrates most prominent in the right lower lobe. A chest CT was ordered to rule out pulmonary embolism but incidentally revealed a spontaneous pneumomediastinum with a small amount of subcutaneous gas in the left lower neck. Additionally, the CT revealed lung opacities characteristic of the initial phases of COVID-19 pneumonia. Cardiothoracic surgery (CTS) was consulted for further evaluation of the spontaneous pneumomediastinum however given that the patient's respiratory status was stable, surgical intervention was deferred. The patient was evaluated by the infectious disease service who recommended plaquenil for 5 days along with ceftriaxone and doxycycline for 7 
days of treatment. Given that the patient never required more than 4 liters of oxygen via nasal cannula and was not noted to be in respiratory distress, corticosteroids and tocilizumab were not administered. The patient was discharged on hospital day 4 on room air along with one additional day of plaquenil and 3 days of doxycycline and ceftin.

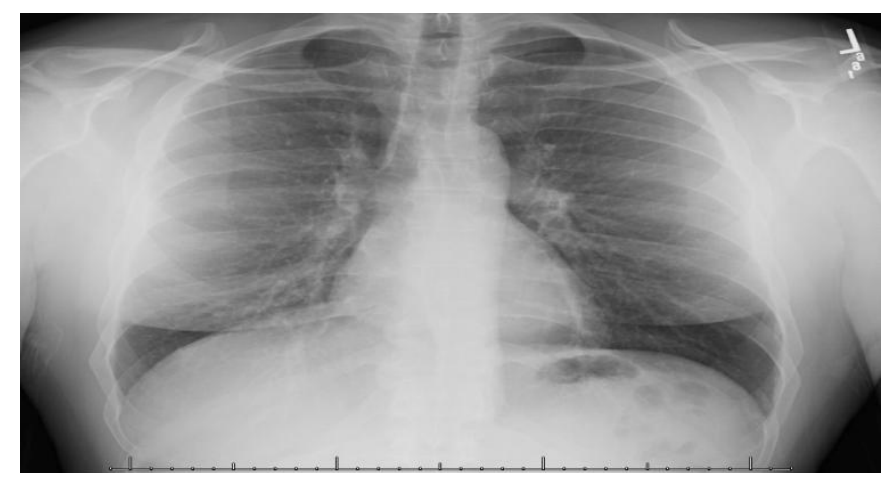

Figure1. Posterior-anterior chest $x$-ray view of patient in case 1 obtained on admission read as bilateral infiltrates, worse in right lower lobe.

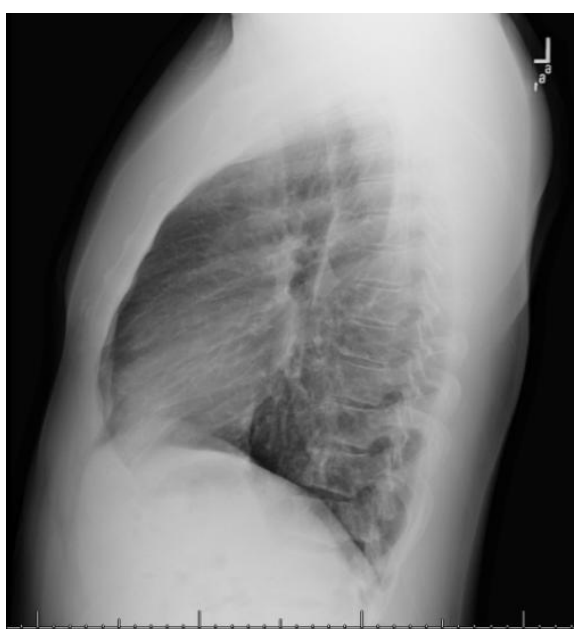

Figure2. Lateral $x$-ray view of patient presented in case 1.

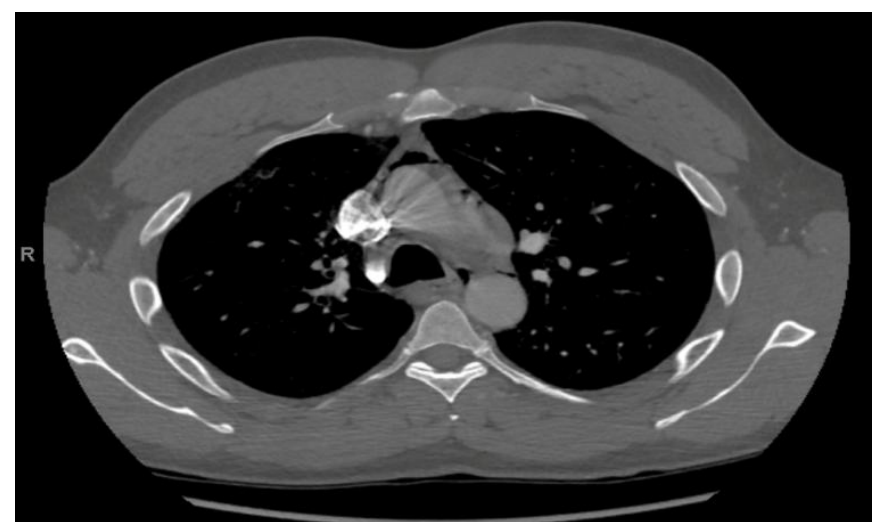

Figure3. Chest computerized tomography (CT) of patient in case 1 revealing a small amount of subcutaneous gas in the left lower neck consistent with pneumomediastinum. Scattered ground-glass opacities are also seen bilaterally.

\subsection{Case 2}

A 65-year-old male with a past medical history for insulin dependent type II diabetes mellitus presented via ambulance for 7 days of worsening dry cough, fevers, chills and myalgias. In the 2 days prior to admission the patient had also been having multiple episodes of non-bloody emesis. Upon arrival to the hospital, vitals were significant for tachycardia (121 beats/min) and tachypnea ( 25 breaths/min). Crepitus of the anterior chest wall was noted on physical examination. A CBC with differential 
was unremarkable for leukocytosis or an elevated absolute neutrophil count, but did reveal an absolute lymphocyte count of 0.40 . Other labs were significant for a ferritin of 1,458, D-dimer 2.78, CRP 15.7, IL-6 371.9, beta hydroxybutyrate 6.42 , serum glucose 843 , anion gap 24 and bicarbonate 15 .

Chest $x$-ray revealed a suspected pneumomediastinum with a small amount of air in the right neck and a subsequent $\mathrm{CT}$ chest confirmed the presence of a moderate volume spontaneous pneumomediastinum surrounding the mid to distal thoracic esophagus which was also present in the right paratracheal and anterior mediastinum. Additionally, CT chest revealed ground-glass opacities in the right lower lobe suggestive of COVID-19 pneumonia. While waiting to be transferred to the intensive care unit (ICU) for management of diabetic ketoacidosis, the patient was noted to appear lethargic, unable to protect his airway, and was emergently intubated. Although initially the patient was found to be negative for COVID-19, a repeat test was found to be positive on hospital day 3. While admitted to the ICU, the patient was treated with intravenous insulin and fluids initially and was then transitioned to subcutaneous insulin. For treatment of COVID19 pneumonia, he was placed on doxycycline and ceftriaxone on admission, however respiratory and blood cultures demonstrated growth of methicillin sensitive staphylococcus aureus (MSSA) and the patient was subsequently started on cefazolin for a total of 7 weeks. Of note, a transthoracic echocardiogram done was negative for vegetation. On hospital day 2 , he received $400 \mathrm{mg}$ of tocilizumab and was started on methylprednisolone $40 \mathrm{mg}$ bid. for a total of 5 days. Repeat imaging on hospital day 4 showed spontaneous resolution of the pneumomediastinum and persistent patchy bilateral airspace opacities. Additionally, the patient was extubated on day 4 to high flow nasal cannula. Although obtaining a gastrograffin study had been initially discussed, a decision was made to defer this as the patient was able to swallow without any difficulty after extubation. He was discharged to inpatient rehabilitation on hospital day 23 on room air.

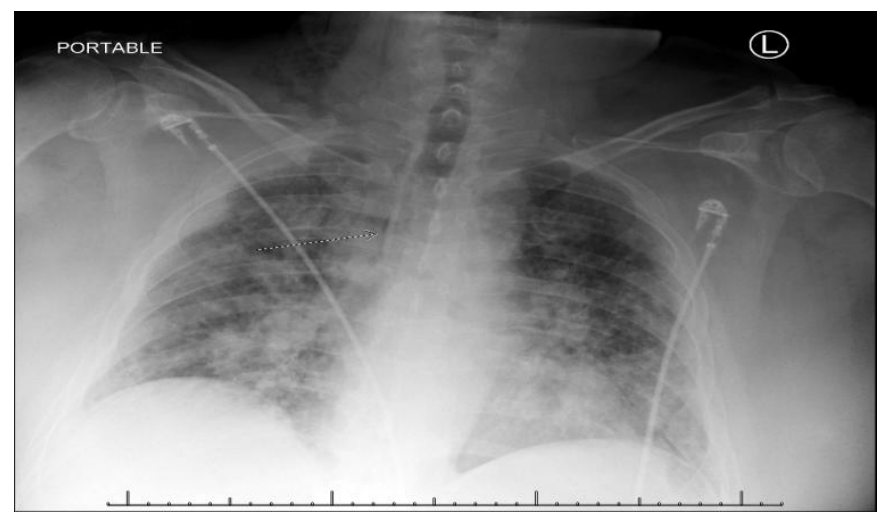

Figure4. Posterior-anterior chest $x$-ray of patient presented in case 2. Linear lucency in the right paratracheal region is present, consistent with pneumomediastinum. Small amount of soft tissue air in the right neck as well. Bilateral interstitial and alveolar opacities.

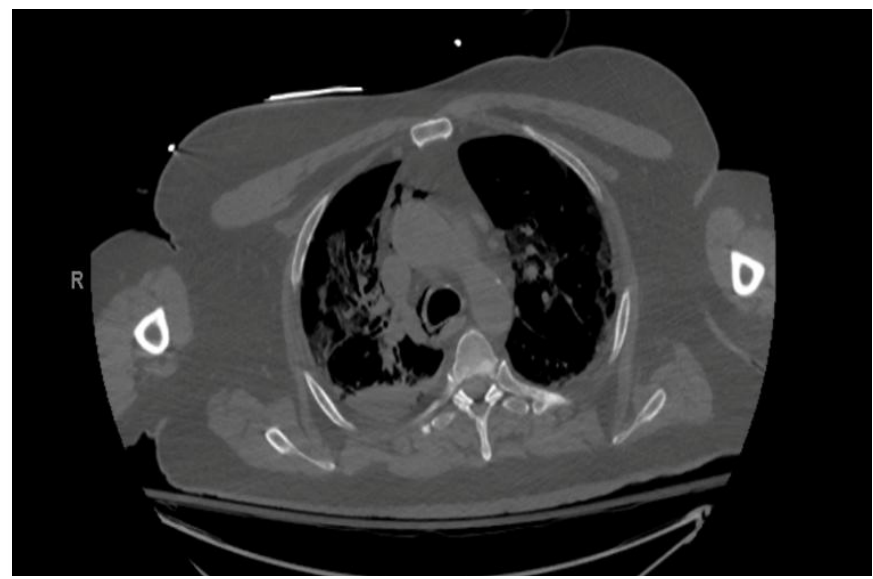

Figure5. Chest CT obtained of patient presented in case 2. Moderate volume pneumomediastinum surrounding the mid to distal thoracic esophagus as well as right paratracheal region and anterior mediastinum is seen. 


\section{DISCUSSION}

Much is yet to be learned about the novel SARS-CoV-2 virus responsible for COVID-19. It is believed that the virus is likely spread via direct contact and respiratory droplets [4]. The most common manifestation of infection is pneumonia characterized by fever, nonproductive cough, and shortness of breath [4,5]. In the early stages of disease, a chest $x$-ray may show an ill-defined consolidation in the lower lobes or may even be unremarkable. However, as the disease progresses, peripheral, bilateral, lower-zone infiltrates usually become prominent [2]. Chest CT is more sensitive with highlighting the radiographic features of COVID-19 and often times demonstrates diffuse ground-glass opacities [3]. Spontaneous pneumomediastinum as seen in our two patients is an extremely rare radiographic feature of COVID-19 with only a handful of cases reported at present.

Pneumomediastinum is defined as the presence of free air in the mediastinum [6]. Categorized into two broad categories, spontaneous or secondary, a pneumomediastinum is thought to occur due to an increase in intrathoracic pressure from alveolar rupture which then allows for air dissection into the mediastinum $[7,8,9]$. It generally presents as a triad of chest pain, dyspnea, and the presence of subcutaneous emphysema on imaging [8,9].

Spontaneous pneumomediastinum is thought to occur without any clearly defined precipitating factor whereas secondary pneumomediastinum is thought to occur as a result of blunt thoracic trauma, esophageal or endobronchial procedures, head and neck surgery, or from gasproducing organisms in the chest cavity $[8,9]$. Spontaneous pneumomediastinum follows a benign, self-limiting course and tends to occur more frequently in young males $[8,9]$. It has been noted to have an incidence of approximately 1 in 44,611 patients treated in an emergency department [9]. Diagnosis is confirmed with poster-anterior and lateral chest $\mathrm{x}$-rays with the most common radiographic feature being radiolucent streaks of gas that highlight the mediastinal structures; chest CT should be reserved for those patients in which the diagnosis is unclear as was the case with the patient presented in case $1[9,10]$.

By destroying type 1 and type II pneumocytes, SARS-CoV-2 induces diffuse alveolar damage which likely then causes the formation of a pneumomediastinum [11]. The first presented patient had no precipitating factors for pneumomediastinum formation other than being infected with SARS-CoV-2 itself. In the case of the second patient, although likely again from the pathogenesis of the virus itself, emesis induced by diabetic ketoacidosis may have a contributing factor for the formation of the pneumomediastinum.

Treatment of spontaneous pneumomediastinum is conservative in nature and includes analgesia, rest, and therapeutic oxygen [9], with most patients recovering with time. Although the association with COVID-19 does not warrant specific therapy in it of itself, the presence of pneumomediastinum on imaging may be an indicator of impending intubation and mortality, and patients should therefore be closely monitored.

\section{ACKNOWLEDGEMENTS}

We would like to thank all the healthcare providers who helped care for these patients.

\section{REFERENCES}

[1] Situation report - 139, Coronavirus disease 2019, June 7 2020. World Health Organization.

[2] Wong HYF, Lam HYS, Fong AH, Leung ST, Chin TW, Yen CSY et. Al. Frequency and Distribution of Chest Radiographic Findings in COVID-19 Positive Patients. Radiology. 2020;201160

[3] Shi H, Han X, Jiang N, Cao Y, Alwalid O, Gu J et. Al. Radiological Findings From 81 Patients with COVID-19 Pneumonia in Wuhan, China: A Descriptive Study. Lancet Infect Dis.2020;20(4):425-434

[4] Guan WJ, Ni ZY, Hu Y, Liang WH, Ou CQ, $\mathrm{He}$ JX et. Al. Clinical Characteristics of Coronavirus Disease 2019 in China. N Engl J Med.2020;382(18):1708-1720

[5] Huang C, Wang Y, Li X, Ren L, Zhao J, Hu Y et. Al. Clinical Features of Patients Infected with 2019 Novel Coronavirus in Wuhan, China. Lancet.2020;395(10223):497-506

[6] Yellin A, Gapany-Gapanavivius M, Lieberman Y. Spontaneous pneumomediastinum; Is it a cause of chest pain? Thorax. 1983;38:383-385.

[7] Macklin MT, Macklin CC. Malignant interstitial emphysema of the lungs and mediastinum as an important occult complication in many respiratory diseases and other conditions: An interpretation of the clinical literature in the light of laboratory experiment. Medicine.1944;23:281-358 
[8] Meireles J, Neves S, Castro A et. Al. Spontaneous pneumomediastinum revisited. Respiratory Medicine CME.2011;4(4):181-183

[9] Macia I, Moya J, Ramos R, Morera R, Escobar I, Saumench $\mathbf{J}$ et. Al. Spontaneous pneumomediastinum: 41 cases. Eur J Cardiothoracic Surg.2007;31(6):1110-1114
[10] Martinez F, Cho Y, Gagliardi J, Razdan R. Spontaneous pneumomediastinum (SP). Appl Radiol. 2011.

[11] Chu CM, Leung YY, Hui JYH, Hung IFN, Chan VL, Leung WS et. Al. Spontaneous Pneumomediastinum in Patients with Severe Acute Respiratory Syndrome. Eur Respir J.2004;23(6):802-4

Citation: Swetha Ramireddy, Saumeet Shah, Fayzan Ahmad, Thomas Bebekoski, Anuradha Sreenivasan, Kevin Brody. Spontaneous Pneumomediastinum: A Rare Radiologic Finding in Covid-19. ARC Journal of Clinical Case Reports. 2020; 6(3):1-5. doi:dx.doi.org/ 10.20431/2455-9806.0603001.

Copyright: (C) 2020 Authors. This is an open-access article distributed under the terms of the Creative Commons Attribution License, which permits unrestricted use, distribution, and reproduction in any medium, provided the original author and source are credited. 